ISSN 0975-3303

Mapana J Sci, 11, 4(2012), 15-42

https:/ / doi.org/ 10.12725/ mjs.23.2

\title{
$L(2,1)-$ Labelling of Cactus Graphs
}

\author{
Nasreen Khan, ${ }^{*}$ Madhumangal Pal† and Anita Pal $\ddagger$
}

\begin{abstract}
The $L(2,1)$-labelling of a graph $G$ is an abstraction of assigning integer frequencies to radio transmitters such that the transmitters that are one unit of distance apart receive frequencies that differ by at least two, and transmitters that are two units of distance apart receive frequencies that differ by at least one. The span of an $L(2,1)$-labelling is the difference between the largest and the smallest assigned frequency. The $L(2,1)$-labelling number of a graph $G$, denoted by $\lambda(G)$, is the least integer $k$ such that $G$ has an $L(2,1)$-labelling of span $k$. A cactus graph is a connected graph in which every block is either an edge or a cycle. The goal of the problem is to show that for a cactus graph $\Delta+1 \leq \lambda(G) \leq \Delta+2$, where $\Delta$ is the degree of $G$. An optimal algorithm is also presented here to label the vertices of cactus graph using $L(2,1)$-labelling technique in $O(n)$ time, where $n$ is the total number of vertices of the cactus graph.
\end{abstract}

* Department of Mathematics, Global Institute of Management and Technology, Krishnagar-741102, West Bengal, India, afsaruddinnkhan@gmail.com

† Department of Applied Mathematics with Oceanology and Computer Programming, Vidyasagar University, Midnapore-721102, West Bengal, India,mmpalvu@gmail.com

‡ Department of Mathematics, National Institute of Technology Durgapur, Durgapur-713209, West Bengal, India, anita.buie@gmail.com

Received: July 2012, Reviewed: Aug. 2012 
Keywords: Graph labelling, $L(h, k)$-labelling, L(2,1)-labelling, cactus graph, frequency assignment, radiocoloring, design of algorithms, analysis of algorithms

\section{Introduction}

Cactus graph is a connected graph in which every block is a cycle or an edge, in other words, no edge belongs to more than one cycle. Cactus graph have extensively studied and used as models for many real world problems. This graph is one of the most useful discrete mathematical structure for modelling problem arising in the real world. It has many applications in various fields like computer scheduling, radio communication system etc. Cactus graph have studied from both theoretical and algorithmic points of view. This graph is a subclass of planar graph and superclass of tree.

Recently $L(2,1)$-labelling problem is attracted by many researchers due to its importance in real life applications. An $L(2,1)$-labelling of a graph $G=(V, E)$ is a function of $f$ from its vertex set $V$ to the set of non-negative integers such that $|f(x)-f(y)| \geq 2$ if $d(x, y)=1$ and $|f(x)-f(y)| \geq 1$ if $d(x, y)=2$, where $d(x, y)$ is the distance between the vertices $x$ and $y$, i.e., the number of edges between $x$ and $y$. The span of an $L(2,1)$-labelling $f$ of $G$ is $\max \{f(v): v \in V\}$. The $L(2,1)$-labelling $\lambda(G)$ of $G$ is the smallest $k$ such that $G$ has a $L(2,1)$-labelling of span $k$. The number $\lambda$ is known as minimum span.

This problem was introduced by Griggs and Yeh [12] (proposed by Roberts) as a variation of channel (frequency) assignment problem, which is stated below.

\subsection{Application of $L(2,1)$-labelling}

The channel assignment problem is to assign a channel (nonnegative integer) to each radio transmitter (radio, T.V, cell phone, etc.) so that interfering transmitters are assigned channels whose separations is not in a set of disallowed separations. Hale [13] formulated this problem into the notion of the T-colouring of a 16 
graph. A variation of the channel assignment problem in which "close" transmitters must receive different channels and "very close" transmitters must receive channels that are at least two channels apart. This problem can be modelled as a graph labelling/ colouring problem where the vertices represent the transmitters two vertices are "very close" if they are adjacent and "close" if they are of distance two in the graph. This type of channel assignment is known in the literature as an $L(2,1)$-labelling.

An $L(2,1)$-labelling of a graph $G$ that uses labels in the set $\{0,1, \ldots . ., k\}$ is called a $k$-labelling. In the context of the channel assignment problem the main aim is to minimize $k$. Griggs and Yeh [12] conjectured that $\lambda(G) \leq \Delta^{2}$, where $\Delta$ represents the maximum degree of a vertex of $G$. Since $L(2,1)$-labelling problem i.e., to determine $\lambda(G)$ is NP-hard [10], the people focuses on verifying Grrigs and Yeh's conjecture and finding exact values for $\Delta$ for particular classes of graphs. A survey of recent literature is given below.

\section{Review of Previous Works}

Several results are known for $L(2,1)$-labelling of graphs, but, to the best of our knowledge no result is known for cactus graph. In this section, the known result for general graphs and some related graphs of cactus graph are presented.

The lower bound for $\lambda(G)$ is $\Delta+1$, which is achieved for the star $K_{1, \Delta}$. Griggs and Yeh [12] prove that $\lambda(G) \leq \Delta^{2}+2 \Delta$ for general graph and improve this upper bound to $\lambda(G) \leq \Delta^{2}+2 \Delta-3$ when $G$ is 3- connected and $\lambda(G) \leq \Delta^{2}$ when $G$ is diameter 2 (diameter 2 graph is a graph where all nodes have either distance 1 or 2 each other). Jonas [15] improves the upper bound to $\lambda(G) \leq \Delta^{2}+2 \Delta-4$ if $\Delta \geq 2$, by constructive labelling schemes. Chang and Kuo [2] further decrease the bound to $\Delta^{2}+\Delta$. Further, Kral and Skrekovski [16] improves this bound $\lambda(G) \leq \Delta^{2}+\Delta-1$ for any graph $G$. The best known result till date is $\lambda(G) \leq \Delta^{2}+\Delta-2$ due to Goncalves [11] 
The problem is simple for paths $P_{n}$ of $n$ vertices. It can easily be verified that $\lambda\left(P_{1}\right)=0, \quad \lambda\left(P_{2}\right)=2, \quad \lambda\left(P_{3}\right)=\lambda\left(P_{4}\right)=3, \quad$ and $\lambda\left(P_{n}\right)=4$ for $n \geq 5$.

When first and last vertices of $P_{n}$ are merged then $P_{n}$ becomes $C_{n-1}$ . In [12], Griggs and Yeh shown that $\lambda\left(C_{n}\right)=4$ for any $n$.

The wheel $W_{n}$, is obtained by joining $C_{n}$ and $K_{1}$, i.e., $W_{n}=C_{n}+K_{1}$. In [23], Yeh shown that $\lambda\left(W_{n}\right)=n+1$.

For the complete graph $K_{n}$ and Kurotoski graph $K_{n, m}$ it can be shown that $\lambda\left(K_{n}\right)=2 n-2, n \geq 1$ and $\lambda\left(K_{n, m}\right)=m+n$.

For any tree $T$, Griggs and Yeh [12] shown that $\lambda(T)$ is either $\Delta+1$ or $\Delta+2$. Heuvel and Mc Guinness prove that $\lambda(G) \leq 2 \Delta+35$ [14] for planar graph. Molloy and Salavatipour [18] reduced this upper bound to $5 \Delta / 3+90$. Wang and Lih [21] proved that if $G$ is a planar graph of girth (girth is defined to be the length of a shortest cycle in $G$ ) at least 5 , then $\lambda(G)=\Delta+21$.

The $L(2,1)$-labelling for chordal graphs has been first investigate by Sakai [20] and he proved that $\lambda(G)=\frac{(\Delta+3)^{2}}{4}$. For unit interval graph (a subclass of chordal graph) he shown that $\lambda(G) \leq 2(\Delta+1)$. Adams et al. [1], give different bounds for certain generalized petersen graphs. A study on $L(d, 1)$-labelling of cartesian product of a cycle and a path is done by Chiang and Yan [4].

For further studies on the $L(2,1)$-labelling, see $[3,10,7,9,10,12,17$, 20].

\section{Preliminaries}

In this section, some basic results are presented. Let $G=\left(V_{G}, E_{G}\right)$ and $H=\left(V_{H}, E_{H}\right)$ be two graphs with disjoin vertex sets. The union of a graph $G$ and $H$, denoted by $G \bigcup H$, is the graph 
whose vertex set is $V_{G} \bigcup V_{H}$ and edge set is $E_{G} \bigcup E_{H}$. The join of $G$ and $H$, denoted by $G+H$, is the graph obtained from $G \bigcup H$ by adding all edges between vertices in $V_{G}$ and $V_{H}$, i.e., $V_{G+H}=V_{G} \bigcup V_{H}$ and $E_{G+H}=E_{G} \bigcup E_{H} \bigcup\left\{(u, v): u \in V_{G} \& v \in V_{H}\right\}$.

The following important lemmas are useful to establish the lower bounds of $\lambda(G)$.

Lemma 1 [2] If $H$ is a subgraph of $G$ then $\lambda(H) \leq \lambda(G)$.

Lemma 2 [2] If $V_{G} \bigcap V_{H}=\phi$ then $\lambda(G \bigcup H)=\max \{\lambda(G), \lambda(H)\}$ and $\lambda(G+H)=\max \left\{\left|V_{H}\right|-1, \lambda(G)\right\}+\max \left\{\left|V_{H}\right|-1, \lambda(H)\right\}+2$.

A particular type of graph union denoted by $\bigcup_{v}$ is defined as follows.

Let $G=\left(V_{G}, E_{G}\right)$ and $H=\left(H_{G}, E_{G}\right)$ be two graphs having only one common vertex $v$, i.e., $V_{G} \bigcap V_{H}=\{v\}$. Let $F=G \bigcup_{v} H$, where $V_{F}=V_{G} \bigcup V_{H}$ and $E_{F}=E_{G} \bigcup E_{H}$. It is easy to verify that $\left|V_{F}\right|=\left|V_{G}\right|+\left|V_{H}\right|-1$ and $\left|E_{F}\right|=\left|E_{G}\right|+\left|E_{H}\right|$. Also, the graphs $G$ and $H$ both are subgraphs of $G \bigcup_{v} H$. We refer this union as v-union.

By Lemma 1, $\lambda(G) \leq \lambda\left(G \bigcup_{v} H\right)$ and $\lambda(H) \leq \lambda\left(G \bigcup_{v} H\right)$. Thus we conclude the following result.

Lemma $3 \lambda\left(G \bigcup_{v} H\right) \geq \max \{\lambda(G), \lambda(H)\}$, where $\{v\}=V_{G} \bigcap V_{H}$.

\section{The $L(2,1)$-labelling of Induced Subgraphs of Cactus Graphs}

Let $G=(V, E)$ be a given graph and subset $U$ of $V$ the induced subgraph by $U$, denoted by $G[U]$, is the given graph $G^{\prime}=\left(U, E^{\prime}\right)$, where $E^{\prime}=\{(u, v): u, v \in U$ and $(u, v) \in E\}$. 


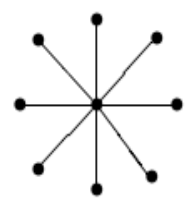

(b) A star $\left(K_{1, \Delta}\right)$

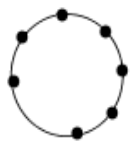

(c) A cycle $\left(C_{n}\right)$

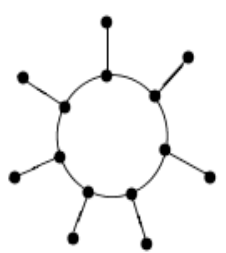

(d) $\mathrm{A} \operatorname{sun}\left(S_{n}\right)$

Figure 1: Induced subgraphs of cactus graph.

The cactus graph is a subclass of planar graph and superclass of tree. From the result of [12] and [4] we can conclude the following.

Lemma 4 For any cactus graph $G, \Delta+1 \leq \lambda(G) \leq 2 \Delta+35$.

The cactus graph have many interested subgraphs, those are illustrated below. An edge is a nothing but $P_{2}$, so $\lambda($ an edge $)=2$. The star graph $K_{1, \Delta}$ is a subgraph of $K_{n, m}$ therefore one can conclude the following result.

Lemma 5 For any star graph $K_{1, \Delta}, \lambda\left(K_{1, \Delta}\right)=\Delta+1$, which is equal to $n$, where $n$ is the number of vertices.

In [12], Griggs and Yeh have label $C_{n}$ by $L(2,1)$-labelling and they have obtained the following result. Here we have given a constructive prove of this result.

Lemma 6 [12] For any cycle $C_{n}$ of length $n, \lambda\left(C_{n}\right)=4=\Delta+2$.

Proof. Let $C_{n}$ be a cycle of length $n$. We classify $C_{n}$ into three groups, viz., $C_{3 k}, C_{3 k+1}, C_{3 k+2}$. The $L(2,1)$-labelling schemes of $C_{3 k}$ are same for any $k$. Similarly for $C_{3 k+1}, C_{3 k+2}$.

Let $v_{0}, v_{1}, v_{2}, \ldots, v_{n-1}$ be the vertices of $C_{n}$. Then the labelling process are as follows.

Case 1. Let $n=3 k \equiv 0(\bmod 3)$, i.e., $C_{3 k}$. 

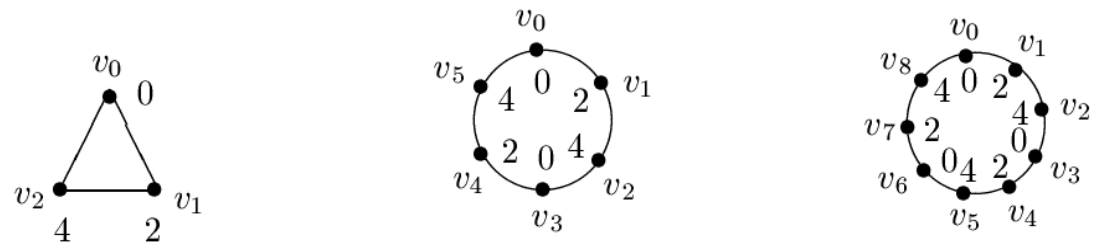

Figure 2: Cases of $C_{3 k}$.

$$
f\left(v_{i}\right)= \begin{cases}0, & \text { if } i \equiv 0(\bmod 3) \\ 2, & \text { if } i \equiv 1(\bmod 3) \\ 4, & \text { if } i \equiv 2(\bmod 3)\end{cases}
$$

Case 2. Let $n=3 k+1 \equiv 1(\bmod 3)$, i.e., $C_{3 k+1}$.
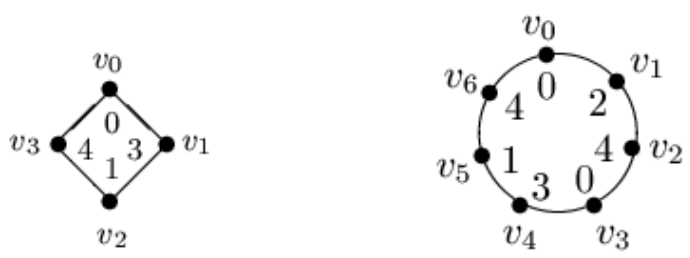

Figure 3: Cases of $C_{3 k+1}$

In this case, the labelling of first $3 k-3$ vertices $v_{0}, v_{1}, v_{2}, \ldots, v_{3 k-3}=v_{n-5}$ are same as in Case 1. For the last four vertices, viz., $v_{n-4}, v_{n-3}, v_{n-2}$ and $v_{n-1}$ the $f$ is redefined as $f\left(v_{n-4}\right)=0, f\left(v_{n-3}\right)=3, f\left(v_{n-2}\right)=1$ and $f\left(v_{n-1}\right)=4$ respectively.

Case 3. Let $n=3 k+2 \equiv 2(\bmod 3)$, i.e., $C_{3 k+2}$.
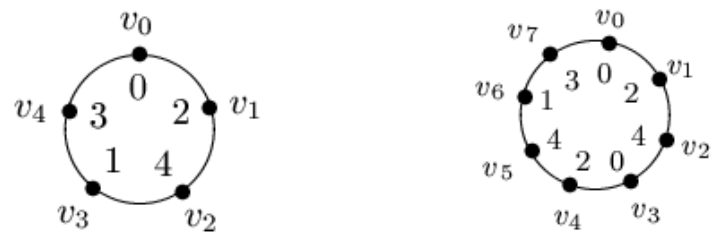

Figure 4: Cases of $C_{3 k+2}$ 
In this case the labelling procedure of the vertices $v_{0}, v_{1}, \ldots, v_{n-3}$ are same as the labelling process of $v_{0}, v_{1}, \ldots, v_{n-1}$ of $C_{3 k}$ in case 1 . Then we label remaining two vertices $v_{n-2}$ and $v_{n-1}$ as $f\left(v_{n-2}\right)=1$ and $f\left(v_{n-1}\right)=3$.

Thus, from three cases, it follows that $\lambda\left(C_{n}\right)=4$.

Let us consider the sun $S_{2 n}$ of $2 n$ vertices. This graph is obtained by adding an edge to each vertex of a cycle $C_{n}$. So $C_{n}$ is a subgraph of $S_{2 n}$. Now the question is what is the value of $\lambda\left(S_{2 n}\right)$ ? Griggs and Yeh [12] prove the following result for the graph similar to $S_{2 n}$

Lemma 7 [12] If a graph $G$ contains three vertices with maximum degree $\Delta \geq 2$ and one of them is adjacent to the other two vertices, then $\lambda(G)$ is at least $\Delta+2$.

For $S_{2 n}, \Delta=3$ hence by Lemma 7, $\lambda\left(S_{2 n}\right) \geq 5$. Here we shall show that $\lambda\left(S_{2 n}\right)=5$.

Lemma 8 For any sun $S_{2 n}, \lambda\left(S_{2 n}\right)=5=\Delta+2$.

Proof. Let $S_{2 n}$ be constructed from $C_{n}$ by adding an edge to each vertex. To label this graph we consider the three cases.

Let $v_{0}, v_{1}, v_{2}, \ldots, v_{n-1}$ be the vertices of $C_{n}$ and $v_{i}$ is adjacent to $v_{i+1}$ and $v_{n-1}$ is adjacent to $v_{0}$. To compute $S_{2 n}$, we add an edge $\left(v_{i}, v_{i}^{\prime}\right)$ to the vertex $v_{i}$, i.e., $v_{i}^{\prime}$ are the pendent vertices. We first label $C_{n}$ by using Lemma 6 . Then we label the pendent vertices as follows.

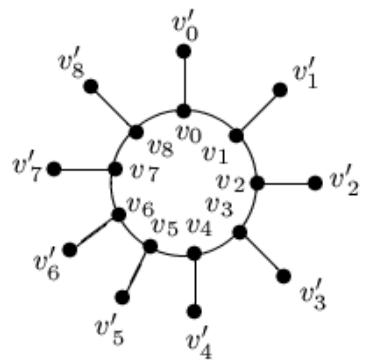


Case 1. Let $n=3 k \equiv 0(\bmod 3)$.

The labelling of $v_{i}^{\prime}$ is assign as $f\left(v_{i}^{\prime}\right)= \begin{cases}3, & \text { if } i \equiv 0(\bmod 3) \\ 5, & \text { if } i \equiv 1(\bmod 3) \\ 1, & \text { if } i \equiv 2(\bmod 3)\end{cases}$

Case 2. Let $n=3 k+1 \equiv 1(\bmod 3)$.

The labelling scheme of $v_{0}^{\prime}, v_{1}^{\prime}, \ldots, v_{n-5}^{\prime}$ are same as the vertices $v_{i}^{\prime}$; $i=0,1, \ldots, n-1$ as in case 1 . Now for the last four pendent vertices $v_{n-4}^{\prime}, v_{n-3}^{\prime}, v_{n-2}^{\prime}, v_{n-1}^{\prime}$ the $f$ are defined as $f\left(v_{n-4}^{\prime}\right)=2, i=n-4, n-1$ and $f\left(v_{i}^{\prime}\right)=5$, for $i=n-3, n-2$ respectively.

Case 3. Let $n=3 k+2 \equiv 2(\bmod 3)$.

Here we label the vertices $v_{3}^{\prime}, v_{4}^{\prime}, \ldots, v_{n-3}^{\prime}$ as

$$
f\left(v_{i}^{\prime}\right)= \begin{cases}3, & \text { if } i \equiv 0(\bmod 3) \\ 5, & \text { if } i \equiv 1(\bmod 3) \\ 0, & \text { if } i \equiv 2(\bmod 3) .\end{cases}
$$

The $f$ values of the vertices $v_{0}^{\prime}, v_{1}^{\prime}, v_{2}^{\prime}, v_{n-2}^{\prime}$ and $v_{n-1}^{\prime}$ are $f\left(v_{0}^{\prime}\right)=4$, $f\left(v_{2}^{\prime}\right)=1$ and $f\left(v_{i}^{\prime}\right)=5$, for $i=1, n-2, n-1$ respectively.

Hence $\lambda\left(S_{2 n}\right)=5=\Delta+2$.

Corollary 1 A graph $G$ contains a cycle $C_{n}$ of length $n$. If two adjacent vertices $v_{0}, v_{n-1}$ of $C_{n}$ have two edges then for $n \equiv 0(\bmod 3)$ and $n \equiv 1(\bmod 3), \lambda(G)=\Delta+1$.

Lemma 9 Let $G$ be a graph obtained from $S_{2 n}$ by adding an edge to each of the pendent vertex of $S_{2 n}$, then $\lambda\left(S_{2 n}\right)=\lambda(G)=\Delta+2=5$.

Proof. According to the previous lemma let the graph be obtained by joining an edge $\left(v_{i}^{\prime}, v_{i}^{\prime \prime}\right)$ to each of the pendent vertex $v_{i}^{\prime}$. Then 
$v_{i}^{\prime \prime}$ 's are the pendent vertices of the new graph. Here we have to label only the pendent vertices. To label the vertices $v_{i}^{\prime \prime \prime}$ s we consider the following three cases.

Case 1. For $n \equiv 0(\bmod 3)$. $f\left(v_{3 i+2}^{\prime \prime}\right)=3$, for $i=0,1, \ldots, \frac{n-3}{3}$ and $f\left(v_{i}^{\prime \prime}\right)=1$, for other vertices.

Case 2. For $n \equiv 1(\bmod 3)$. $f\left(v_{3 i+2}^{\prime \prime}\right)=3$, for $i=0,1, \ldots, \frac{n-7}{3}, \quad f\left(v_{n-4}^{\prime \prime}\right)=4, \quad f\left(v_{i}^{\prime \prime}\right)=0$, for $i=n-3, n-2, n-1$ and $f\left(v_{i}^{\prime \prime}\right)=1$, for other vertices.

Case 3. For $n \equiv 2(\bmod 3)$. $f\left(v_{3 i+2}^{\prime \prime}\right)=3$, for $i=0,1, \ldots, \frac{n-5}{3}, f\left(v_{i}^{\prime \prime}\right)=0$, for $i=n-2, n-1$ and $f\left(v_{i}^{\prime \prime}\right)=1$, for other vertices.

So, $\lambda(G)=5=\Delta+2$.

Corollary 2 Let $G$ be a graph obtained from $C_{n}$ by adding $P_{i}, \quad i=1,2,3, \ldots$ to one or more vertices of $C_{n}$, then $\lambda(G)=\lambda\left(S_{2 n}\right)=\Delta+2=5$.

Lemma 10 Let $G=C_{n} \bigcup_{v_{0}} C_{m}$ then $\lambda\left(C_{n} \bigcup_{v_{0}} C_{m}\right)=5=\Delta+1$, where $\Delta$ is the degree of the common vertex $v_{0}$.

Proof. Let $C_{n}$ and $C_{m}$ be two cycles of $G$. Let $v_{0}, v_{1}, \ldots, v_{n-1}$ be the vertices of $C_{n}$ such that $v_{i}$ is adjacent to $v_{i+1}, 0 \leq i \leq n-2$ and $v_{0}$ is adjacent to $v_{n-1}$. The label $f\left(v_{i}\right)$ of $v_{i} \in C_{n}$ is assign as in Lemma 6.

Again let $v_{0}, v_{1}^{\prime}, \ldots, v_{m-1}^{\prime}$ be the vertices of $C_{m}$ such that $v_{0}$ is adjacent to $v_{1}^{\prime}$ and $v_{m-1}^{\prime}$. Also, $v_{i}^{\prime}$ is adjacent to $v_{i+1}^{\prime}, 0 \leq i \leq m-2$. 
At the time of labelling of the vertices of $C_{n}$, we assign the label of $v_{0}$ to 0 , i.e., $f\left(v_{0}\right)=0$.

Now, we assign the labels to the vertices of $C_{m}$ by considering three cases, viz., $m=3 k, m=3 k+1$ and $m=3 k+2$.

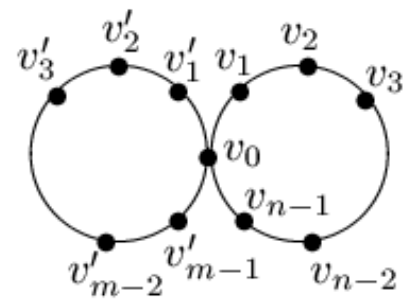

Figure 6: Illustration of Lemma 10.

Case 1. If $n \equiv 0(\bmod 3), m \equiv 0(\bmod 3)$.

Here $f\left(v_{0}\right)=0$. Then the labelling of other vertices of $C_{m}$ is as follows:

$$
f\left(v_{1}^{\prime}\right)=3, f\left(v_{2}^{\prime}\right)=5 \text { and } f\left(v_{i}^{\prime}\right)= \begin{cases}0, & \text { if } i \equiv 0(\bmod 3) \\ 3, & \text { if } i \equiv 1(\bmod 3) \\ 5, & \text { if } i \equiv 2(\bmod 3) .\end{cases}
$$

If $n \equiv 0(\bmod 3), m \equiv 1(\bmod 3)$.

Then the label of the vertices $v_{0}, v_{1}^{\prime}, \ldots, v_{m-5}^{\prime}$ are same as given in the above subcase. Now the label of the last four vertices $v_{m-4}^{\prime}, v_{m-3}^{\prime}, v_{m-2}^{\prime}, v_{m-1}^{\prime}$ are $f\left(v_{m-4}^{\prime}\right)=0, f\left(v_{m-3}^{\prime}\right)=3, f\left(v_{m-2}^{\prime}\right)=1$, and $f\left(v_{m-1}^{\prime}\right)=5$ respectively. When $m=4$ then, $f\left(v_{1}^{\prime}\right)=3, f\left(v_{2}^{\prime}\right)=1$ and $f\left(v_{3}^{\prime}\right)=5$. If $n \equiv 0(\bmod 3), m \equiv 2(\bmod 3)$. 
We label the vertices $v_{1}^{\prime}, v_{2}^{\prime}, v_{3}^{\prime}$ and $v_{4}^{\prime}$ as

$f\left(v_{1}^{\prime}\right)=3, f\left(v_{2}^{\prime}\right)=5, f\left(v_{3}^{\prime}\right)=1$ and $f\left(v_{4}^{\prime}\right)=2$.

And other vertices as same process as in above subcase (for $m \equiv 0(\bmod 3))$.

When $m=5$, then we label the graph as

$$
f\left(v_{i}\right)=\left\{\begin{array}{ll}
0, & \text { if } i \equiv 0(\bmod 3) ; \\
3, & \text { if } i \equiv 1(\bmod 3) ; \\
5, & \text { if } i \equiv 2(\bmod 3) ;
\end{array} \text { and } f\left(v_{i}^{\prime}\right)= \begin{cases}2, & \text { if } i=1 ; \\
5, & \text { if } i=2 ; \\
1, & \text { if } i=3 ; \\
4, & \text { if } i=4\end{cases}\right.
$$

Case 2. If $n \equiv 1(\bmod 3), m \equiv 1(\bmod 3)$.

The labelling scheme of the vertices of $C_{m}$ are same as in case 1 (for $m \equiv 1(\bmod 3))$.

When $n=4$ and $m=4$, then

$$
f\left(v_{i}\right)=\left\{\begin{array}{ll}
0, & \text { if } i=0 ; \\
3, & \text { if } i=1 ; \\
5, & \text { if } i=2 ; \\
2, & \text { if } i=3 ;
\end{array} \text { and } f\left(v_{i}^{\prime}\right)= \begin{cases}4, & \text { if } i=1 \\
1, & \text { if } i=2 \\
5, & \text { if } i=3\end{cases}\right.
$$

If $n \equiv 1(\bmod 3), m \equiv 2(\bmod 3)$.

When $m>5$, the labels of $C_{m}$ are same as in case 1 .

When $m=5$, then label the vertices of $C_{m}$ as same as in case 1 . Now we label the vertices of $C_{n}$ as same as the labelling of $C_{m}$ in case 1 (for $m \equiv 1(\bmod 3))$, except the labelling of the vertices $v_{n-2}$ and $v_{n-1}$. We label these vertices as $f\left(v_{n-2}\right)=1$ and $f\left(v_{n-1}\right)=5$ respectively.

Case 3. If $n \equiv 2(\bmod 3), m \equiv 0(\bmod 3)$. 
For the vertices $v_{4}^{\prime}, v_{5}^{\prime}, \ldots, v_{m-3}^{\prime}$ the labelling procedure is

$$
f\left(v_{i}^{\prime}\right)= \begin{cases}0, & \text { if } i \equiv 1(\bmod 3) ; \\ 3, & \text { if } i \equiv 2(\bmod 3) \\ 5, & \text { if } i \equiv 0(\bmod 3)\end{cases}
$$

Then we label other vertices as

$$
f\left(v_{1}^{\prime}\right)=4, f\left(v_{2}^{\prime}\right)=1, f\left(v_{3}^{\prime}\right)=5, f\left(v_{m-2}\right)=1 \text { and } f\left(v_{m-1}\right)=5 .
$$

When $n=5$ and $m=3$, then we label the vertices as

$$
f\left(v_{0}\right)=0, f\left(v_{1}\right)=2, f\left(v_{2}\right)=4, f\left(v_{3}\right)=1, f\left(v_{4}\right)=5,
$$

and $f\left(v_{1}^{\prime}\right)=3, f\left(v_{2}^{\prime}\right)=5, f\left(v_{3}^{\prime}\right)=1, f\left(v_{4}^{\prime}\right)=4$.

Therefore, $\lambda(G)=\lambda\left(C_{n} \bigcup_{v_{0}} C_{m}\right)=5=\Delta+1$.

Some times a cycle $C_{3}$ of length 3 is called a triangle. A triangle may be a subgraph of a cactus graph. Also, a triangle shape star, (i.e., all the triangles have a common cutvertex) be a subgraph of a cactus graph. Let $T_{0}, T_{1}, \ldots, T_{n-1}$ be the $n$ triangles meet at a common cutvertex $v_{0}$ and we denote this graph by $G$, which is equivalent to $\bigcup_{v_{0}} T_{i}$. The number of vertices and edges of $G$ are $2 n+1$ and $3 n$ respectively.

Lemma 11 Let a graph $G$ contains finite number of cycles of finite lengths with a common cutvertex. Then $\lambda(G)=\Delta+2$, when $G$ contains odd number triangles and $\Delta+1$, for other cases, where $\Delta$ is the degree of the cutvertex.

Proof. First we prove that if the graph contains $n$ triangles then $\lambda(G)=\Delta+1$ or $\Delta+2$ according as $n$ is even or odd. Let us denote the $n$ such triangles by $T_{0}, T_{1}, T_{2}, \ldots, T_{n-1}$ (shown in Figure 6). Let $v_{0}$ be the common cutvertex. If $\Delta$ be its degree, then $\Delta=2 n$. Let $v_{0}$, $v_{i 1}$ and $v_{i 2}$ be the vertices of $T_{i}$. We label $v_{0}$ by 0 . 
Nasreen Khan, Madhumangal Pal and Anita Pal ISSN 0975-3303

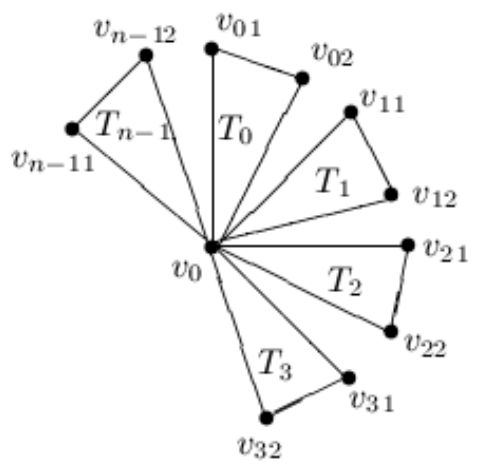

Figure 7: A graph contains $n$ triangles

Then the labels of $T_{i}$ 's are as follows:

If $i$ is even then, $f\left(v_{i j}\right)= \begin{cases}2 i+2, & \text { for } j=1 ; \\ 2 i+4, & \text { for } j=2 ;\end{cases}$

and if $i$ is odd then, $f\left(v_{i j}\right)= \begin{cases}2 i+1, & \text { for } j=1 ; \\ 2 i+3, & \text { for } j=2 .\end{cases}$

If $n$ is even then let $n=2 k$ for some $k$. The label of the vertex $v_{2 k-1,2}$ of the cycle $T_{2 k-1}$ is $f\left(v_{2 k-1,2}\right)=4 k+1=2 n+1=\Delta+1$.

And if $n$ is odd then $n=2 k+1$. Then the labels of the vertex $v_{2 k, 2}$ of the cycle $T_{2 k}$ is $f\left(v_{2 k, 2}\right)=4 k+4=2 n+2=\Delta+2$. 


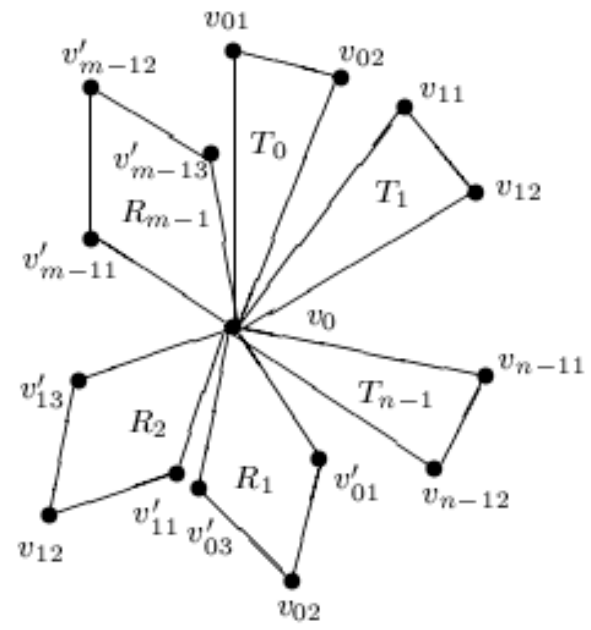

Figure 8: The graph contains $n$ triangles and $m$ cycles of lengths 4

Now if we prove for a graph which contains $n$ number of cycles of length 3 and $m$ number of cycles of length $4, \lambda(G)=\Delta+1$, then the result is true for general case. Let the graph contains $T_{0}, T_{1}, \ldots$, $T_{n-1}$ be the $n$ number of cycles of length 3 and $R_{0}, R_{1}, \ldots, R_{m-1}$ be the $m$ number of cycles of length 4 (shown in Figure 7). All cycles are joined with $v_{0}$. Then $\Delta=2 n+2 m$. Again let $v_{0}, v_{i j} ; j=1,2$ be the vertices of $T_{i}$ 's and $v_{0}, v_{j k}^{\prime} ; k=1,2,3$ be the vertices of $R_{j}$ 's. Using the above rule we label the vertices of all $T_{i}$ 's. Here $f\left(v_{0}\right)=0$. Then we label vertices of $R_{j}$ 's are as follows.

If $n$ is even the labelling of the adjacent vertices of $v_{0}$ of $R_{j}$ 's are as

for $j=0,1, \ldots, m-1, f\left(v_{j k}^{\prime}\right)= \begin{cases}2 n+2 j+2, & \text { for } k=1 \text {; } \\ 2 n+2 j+3, & \text { for } k=3 .\end{cases}$

If $n$ is odd then we label the adjacent vertices of $v_{0}$ of $R_{j}$ 's as 
for $\quad j=1,2, \ldots, m-1, \quad f\left(v_{j k}^{\prime}\right)=\left\{\begin{array}{ll}2 n+2 j+2, & \text { for } k=1 ; \\ 2 n+2 j+3, & \text { for } k=3 ;\end{array}\right.$ and $f\left(v_{0 k}^{\prime}\right)= \begin{cases}2 n+1, & \text { for } k=1 \\ 2 n+3, & \text { for } k=3\end{cases}$

Other vertices of $R_{j}$ 's as $f\left(v_{j 2}^{\prime}\right)=1$, for $j=0,1, \ldots, m-1$.

So we get $f\left(v_{m-1,3}^{\prime}\right)=2 n+2(m-1)+3=2 n+2 m+1=\Delta+1$.

Hence the lemma is proved.

Corollary 3 If a graph $G$ contains finite number of cycles of any length (except odd number of cycles of length three) with a common cutvertex, then $\lambda(G)=\Delta+1$, where $\Delta$ be the degree of the cutvertex.

Lemma 12 Let $G$ be a graph which contains finite number of cycles of any length and finite number of edges. If $v_{0}$ be the common cutvertex with degree $\Delta$ then $\lambda(G)=\Delta+1$.

Proof. If we prove that for a graph $G$ contains $n$ number of cycles of length $3, m$ number of cycles of length 4 and $p$ number of edges, $\lambda(G)=\Delta+1$, then generally we can say that the above statement is true.

According to the previous lemma let $v_{k}^{\prime} ; k=0,1, \ldots, p-1$ be the end vertices of the edges joined with the common cutvertex $v_{0}$. Here $\Delta=2 n+2 m+p$. Now we label the end vertices by $L(2,1)$-labelling as follows.

$f\left(v_{k}^{\prime}\right)=2 n+2 m+2+k$, for $k=0,1, \ldots, p-1$.

Here $f\left(v_{p-1}^{\prime}\right)=2 n+2 m+2+p-1=2 n+2 m+p=\Delta+1$.

So, $\lambda(G)=\Delta+1$.

Corollary 4 When the end vertices of the edges of the graph $G$ have another edges and each vertices of the cycles have another edges, then the value of $\lambda(G)$ remains unchanged. 
Lemma 13 Let $G$ be a graph, contains a cycle of any length and finite number of edges, they have a common cutvertex $v_{0}$. If $\Delta$ be the degree of the cutvertex then, $\lambda(G)=\Delta+1$.

Proof. Let $G$ be a graph which contains a cycle $C_{n}$ of length $n$ and $p$ number of edges. Again let $v_{0}, v_{1}, \ldots, v_{n-1}$ be the vertices of $C_{n}$ and $v_{0}^{\prime}, v_{1}^{\prime} ; v_{2}^{\prime}, \ldots ; v_{p-1}^{\prime}$ are the end vertices of all edges. Here $v_{0}$ is the common cutvertex with degree $\Delta(=2+p)$. Then we label the graph as follows.

Case 1. For $n \equiv 0(\bmod 3)$ and $n \equiv 1(\bmod 3)$.

We first label the vertex $v_{0}$ by 0 . That is, $f\left(v_{0}\right)=0$. Then we label the vertex $v_{0}^{\prime}$ by 3 , i.e., $f\left(v_{0}^{\prime}\right)=3$. And then we label the end vertices of edges as $f\left(v_{i}^{\prime}\right)=4+i, i=1 ; 2, \ldots ; p-1$.

Now $f\left(v_{p-1}^{\prime}\right)=4+(p-1)=3+p=\Delta+1$.

Case 2. For $n \equiv 3(\bmod 3)$.

Here we label the first end vertex of the edge $v_{0}^{\prime}$ by 4 , i.e., $f\left(v_{0}^{\prime}\right)=4$. Then we label the remaining end vertices of the edges as same as given in the above case.

From two cases we see that, $\lambda(G)=\Delta+1$.

Hence the result.

Lemma 14 Let $G$ be a graph contains a cycle of any length and each vertex of the cycle has another cycle of length three. If $\Delta$ is the degree of $G$ then $\lambda(G)=\Delta+3$.

Proof. Let $G$ be a graph which contains a cycle $C_{n}$ of length $n$ and each vertex of $C_{n}$ connect with another cycle of length three. Let $v_{0}, v_{1}, \ldots, v_{n-1}$ be the vertices of $C_{n}$ and $v_{01}, v_{02} ; v_{11}, v_{12} ; \ldots ; v_{n-11}, v_{n-12}$ are the vertices of all $C_{3}$ 's. That is 
Nasreen Khan, Madhumangal Pal and Anita Pal ISSN 0975-3303

$v_{0}, v_{1}, \ldots, v_{n-1}$ are all cut vertices of the graph $G$. First we label the vertices of $C_{n}$ by using Lemma 6 .

We label the graph $G$ as follows.

Case 1. For $n \equiv 0(\bmod 3)$.

If $i \equiv 0(\bmod 3)$, then $f\left(v_{i j}\right)= \begin{cases}3, & \text { for } j=1 ; \\ 5, & \text { for } j=2 .\end{cases}$

If $i \equiv 1(\bmod 3)$, then $f\left(v_{i j}\right)= \begin{cases}5, & \text { for } j=1 ; \\ 7, & \text { for } j=2 .\end{cases}$

If $i \equiv 2(\bmod 3)$, then $f\left(v_{i j}\right)= \begin{cases}1, & \text { for } j=1 \\ 6, & \text { for } j=2 .\end{cases}$

Case 2. For $n \equiv 1(\bmod 3)$.

Here we label the vertices of different cycles of lengths 3 as same as in the above case except the vertices which are adjacent to $v_{n-4}, v_{n-3}, v_{n-2}$ and $v_{n-1}$. Now we label those vertices as

$$
f\left(v_{n-4, j}\right)=\left\{\begin{array}{ll}
2, & \text { for } j=1 ; \\
5, & \text { for } j=2 ;
\end{array} \quad f\left(v_{n-3, j}\right)= \begin{cases}5, & \text { for } j=1 ; \\
7, & \text { for } j=2\end{cases}\right.
$$

$$
\begin{gathered}
f\left(v_{n-2, j}\right)= \begin{cases}5, & \text { for } j=1 ; \\
7, & \text { for } j=2 ;\end{cases} \\
f\left(v_{n-1, j}\right)= \begin{cases}2, & \text { for } j=1 ; \\
6, & \text { for } j=2 .\end{cases}
\end{gathered}
$$


Case 3. For $n \equiv 2(\bmod 3)$.

Now we label the vertices $v_{11}, v_{12} ; v_{21}, v_{22} ; \ldots ; v_{n-41}, v_{n-42}$ as per rule given in case 1 . The label of the vertices $v_{01}, v_{02} ; v_{n-31}, v_{n-32} ; v_{n-21}, v_{n-22} ; v_{n-11}, v_{n-12}$ are as follows.

$$
\begin{gathered}
f\left(v_{0 j}\right)=\left\{\begin{array}{cc}
4, & \text { for } j=1, \\
6, & \text { for } j=2,
\end{array} \quad f\left(v_{n-3, j}\right)= \begin{cases}0, & \text { for } j=1, \\
6, & \text { for } j=2,\end{cases} \right. \\
f\left(v_{n-2, j}\right)= \begin{cases}5, & \text { for } j=1, \\
7, & \text { for } j=2,\end{cases} \\
f\left(v_{n-1, j}\right)= \begin{cases}5, & \text { for } j=1, \\
7, & \text { for } j=2,\end{cases}
\end{gathered}
$$

Thus, from all the above cases, it follows that $\lambda(G)=\Delta+3$. W

Corollary 5 Let $G$ be a graph contains a cycle of any length and each vertex of the cycle has two or many cycles of length three. If $\Delta$ be the degree of $G$ then $\lambda(G)=\Delta+3$.

Corollary 6 Let $G$ be a graph contains a cycle of any length and each vertex of the cycle has another cycle of any length (>3). If $\Delta$ be the degree of $G$ then $\lambda(G)=\Delta+2$.

Lemma 15 Let $G_{1}$ and $G_{2}$ be two cactus graphs. If $\Delta_{1}+1 \leq \lambda\left(G_{1}\right) \leq \Delta_{1}+3 \quad$ and $\quad \Delta_{2}+1 \leq \lambda\left(G_{2}\right) \leq \Delta_{2}+3$, then, $\Delta+1 \leq \lambda(G) \leq \Delta+3$, where $G=G_{1} \bigcup_{v} G_{2}$.

Proof. Let $G_{1}$ and $G_{2}$ be two cactus graphs and $\Delta_{1}, \Delta_{2}$ be the degrees of them. Again let $u$ and $v$ be two vertices of that graphs and $x, y$ be the labels of $u$ and $v$ respectively. If we merge two cactus graphs $G_{1}$ and $G_{2}$ with the vertex $v$ then we get a new 
cactus graphs $G\left(=G_{1} \bigcup_{v} G_{2}\right)$. Let $\Delta$ be the degree of new cactus graph $G$, where $\max \left\{\Delta_{1}, \Delta_{2}\right\} \leq \Delta \leq \Delta_{1}+\Delta_{2}$. For the graph $G_{1}$, $\Delta_{1}+1 \leq \lambda\left(G_{1}\right) \leq \Delta_{1}+3$ and $G_{2}, \Delta_{2}+1 \leq \lambda\left(G_{2}\right) \leq \Delta_{2}+3$. Now we have to prove that the lower and upper bounds of $\lambda$ will preserve for the new cactus graph $G$.

Assume that the label of $u$ be fixed and it be 0 , i.e., $x=0$, and the label $y$ of $v$ lies between 0 to $\Delta_{2}+3$. That is, the label difference between $x$ and $y$ will be $0,1, \ldots, \Delta_{2}+3$.

Let the label of the vertices $u$ and $v$ be same, i.e., $x=y$. If we merge the two cactus graphs, then label of $v$ remains unchanged and the labels of adjacent vertices of $v$ will changed as per the rule of $L(2,1)$-labelling, i.e., the label difference between any two adjacent vertices is at least 2 and any two vertices which are at distance two is at least 1 . If we increase the label numbers by 1 of all vertices of $G_{2}$ except $v$ then there are at least one vertex in which we adjust the labelling to preserve the lower and upper bounds of $\lambda$.

When the label difference between $x$ and $y$ is 1, i.e., $y=x+1$, then without loss of generality we assume that the label numbers of adjacent vertices of $u$ are $x+2$ and $x+3$. And the label numbers of adjacent vertices of $v$ are $x+3$ and $x+4$ respectively. If we merge the two cactus graphs then the labels of $u$ and $v$ will be same. Then we change the label number $x+3$ to $x+5$ to the graph $G_{2}$. If we increase the label numbers of all vertices of $G_{2}$ by 1 except $v$ then we get at least one vertex in which we adjust the labelling to preserve the lower and upper bounds of $\lambda$, i.e., the $\lambda$ value of new cactus graph can't be less than $\Delta+1$ and greater than $\Delta+3$.

Similarly, for the label differences $2,3, \ldots, \Delta_{2}+3$, the lower and upper bounds of $\lambda$ for the new cactus graph will preserve.

Hence the proof. 
The $L(2,1)$-labelling of all subgraphs of cactus graphs and their combinations are discussed in the previous lemmas. From these results we conclude that the $\lambda$-value of any cactus graph can not be more than $\Delta+3$. Hence we have the following theorem.

Theorem 1 If $\Delta$ is the degree of a cactus graph $G$, then $\Delta+1 \leq \lambda(G) \leq \Delta+3$.

Proof. The $L(2,1)$-labelling of all possible subgraphs of cactus graph are discussed and have shown that $\Delta+1 \leq \lambda \leq \Delta+3$. Let $G$ be obtained by $v$-union of two cactus graphs then $G$ becomes a cactus graph and it is proved that $\lambda(G)$ should satisfy the inequality $\Delta+1 \leq \lambda(G) \leq \Delta+3$ (Lemma 15).

Hence the theorem.

\section{The Algorithm and Time Complexity}

\subsection{Construction of an equivalent graph $G^{\prime}$ of $G$}

Using DFS we obtain all blocks and cutvertices of a cactus graph $G=(V, E)$. Let the blocks be $B_{0}, B_{1}, B_{3}, \ldots, B_{N-1}$ and the cut vertices be $C_{0}, C_{1}, \ldots, C_{R-1}$ where $N$ is the total number of blocks and $R$ is the total number of cut vertices.

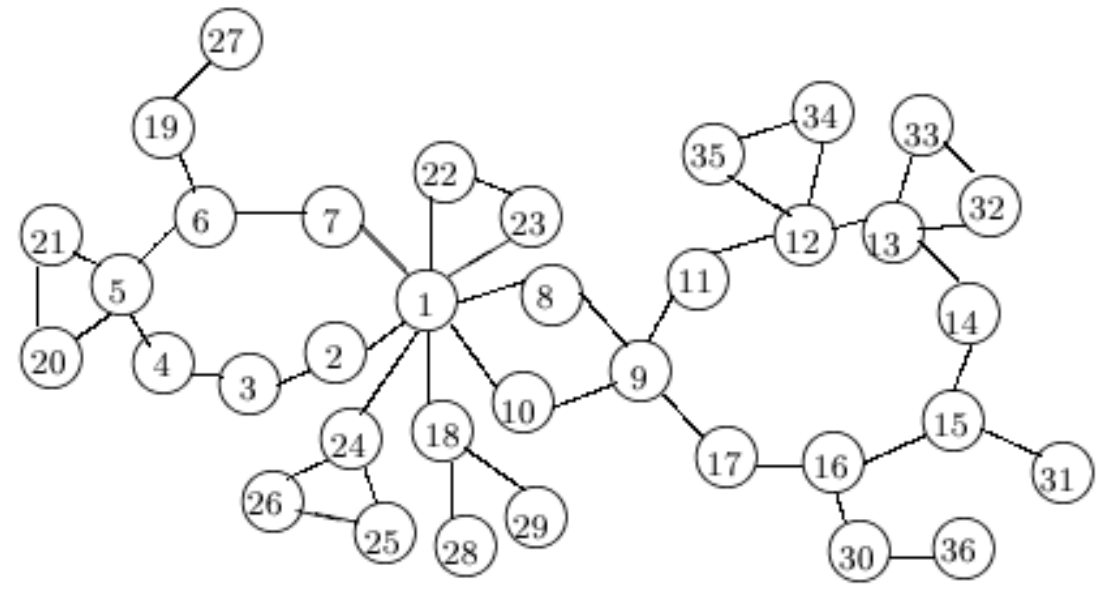

Figure 9: A cactus graph G. 
Nasreen Khan, Madhumangal Pal and Anita Pal ISSN 0975-3303

The blocks of the cactus graph shown in Figure 9 are

$\left\{B_{0}=(1,2,3,4,5,6,7), B_{1}=(1,24), B_{2}=(1,18), B_{3}=(1,8,9,10), B_{4}=(1,22,23)\right.$,

$B_{5}=(6,19), B_{6}=(5,20,21), B_{7}=(24,25,26), B_{8}=(18,28), B_{9}=(18,29)$,

$B_{10}=(9,11,12,13,14,15,16,17), B_{11}=(19,27), B_{12}=(16,30), B_{13}=(15,31)$,

$\left.B_{14}=(13,32,33), B_{15}=(12,34,35), B_{16}=(30,36)\right\}$

and the cutvertices are $C=\{1,5,9,12,13,15,16,18,19,24,30\}$.

\section{Level}

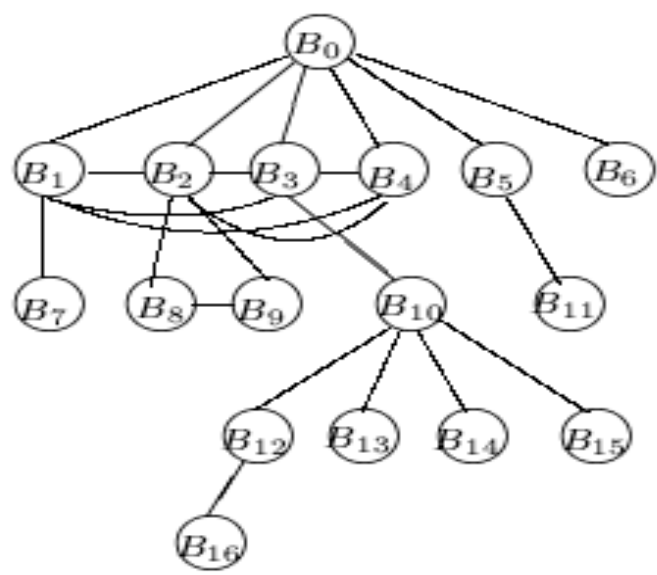

0

1

2

3

4

Figure 10: The equivalent graph $G^{\prime}$ of $G$.

Now we have in a position to construct an equivalent graph $G^{\prime}$ of $G$ whose vertices are the blocks of $G$ and an edge is defined between two blocks if they are adjacent blocks of $G$.

i.e., $G^{\prime}=\left(V^{\prime}, E^{\prime}\right)$ where $V^{\prime}=\left\{B_{0}, B_{1}, \ldots, B_{N-1}\right\}$

and $E^{\prime}=\left\{\left(B_{i}, B_{j}\right): i \neq j, i, j=0,1, \ldots, N-1, B_{i}\right.$ and $B_{j}$ are adjacent blocks $\}$.

The graph $G^{\prime}$ for the graph $G$ of Figure 8 is shown in Figure 9. 


\subsection{Labelling of vertices}

To start the algorithm of $L(2,1)$-labelling of a cactus graph, we first construct a graph $G^{\prime}$ which is equivalent to the given graph $G$. Next we take any arbitrary finite length cycle of any length of $G$ as starting block. The block is so chosen that the degree of the cutvertex is maximum. We denote the start block as $B_{0}$ at level 0 . Now we label the block $B_{0}$ using the Lemma 6 .

Then we label the blocks of level 1 from left to right. The blocks of level 1 say $B_{11}, B_{12}, B_{13}, \ldots$, are either edges or triangles or cycles of finite lengths. We consider the first block $B_{11}$ of level 1 which is adjacent to $B_{0}$. If the block is an edge then label the block by using Lemma 8. If it is cycle then we label it by using Lemma 10. The blocks which are adjacent to $B_{0}$ only at level 1 we label them according to the rule of the block $B_{11}$. Next we consider the second block $B_{12}$. If it is not adjacent to $B_{11}$, then we label the vertices of $B_{12}$ using Lemma 8 or 10 . If it is adjacent to $B_{11}$, then we label the vertices of $B_{12}$ by using the Lemmas 11,12, 13 and 15 . Now we consider the block $B_{1 i}$. If it is not adjacent with any block of level 1, then we label it by Lemmas 8 and 10. But, if it is adjacent at least one block of level 1 then we follow the rules of Lemmas 11, 12, 13 and 15.

Now we label the the vertices of the blocks of level 2 then level 3 and so on as per the procedure mentioned above.

Suppose a block of level $l$, say $B_{l j}$ is an edge and it is adjacent to a block say $B_{l+1, k}$ of level $l+1$ which is also an edge. Then we label the block by using the Lemma 5 .

Suppose a block, say $B_{k-1, i}$ of level $k-1$ is a triangle, its adjacent block at level $k$, say $B_{k j}$, is also a cycle of finite length. If each vertices of the block $B_{k j}$ contains triangles, one of them say $B_{k+1, p}$ of level $k+1$, then we label the block by using the Lemma 14 . 


\section{Algorithm MINLV}

Input: The cactus graph $G=(V, E)$.

Output: Label of its vertices.

Step 1: Compute the blocks and cut vertices of $G$ and construct an equivalent graph $G^{\prime}$ of $G$.

Step 2: Take any arbitrary cycle of any length, say $B_{0}$ as starting block, where the degree of the cut vertex of $B_{0}$ is maximum.

Step 3: We label the block $B_{0}$ using Lemma 6.

Step 4: Consider the blocks $B_{1 j}, j=1,2,3, \ldots$, of level 1 . Label the blocks from left to right as follows.

(i) Take the first block $B_{11}$ which is adjacent to $B_{0}$. If it is an edge then we label $B_{11}$ by using Lemma 8 and if it is cycle then we label it by using Lemma 10.

(ii) Next we consider the second block $B_{12}$. If it is not adjacent to $B_{11}$, then label it by using Lemma 8 or 10 . If $B_{12}$ is adjacent to $B_{11}$, then label it by using Lemmas $11,12,13$ and 15 .

(iii) Consider the block $B_{1 i}$. If it is not adjacent to any block of level 1, then label it by Lemma 8 or 10. But if it is adjacent to at least one block of level 1 then follow the rules of Lemmas 11, 12, 13 and 15.

(iv) The blocks which are adjacent to $B_{0}$ only then label them by the process similar to $B_{11}$.

Step 5: Consider the blocks of subsequent and so on. We label them as per the procedure of step 4 , step 6 and step 7 .

Step 6: Suppose a block of level $l$, say $B_{l j}$ is an edge and another block $B_{l+1, k}$ of level $l+1$ adjacent to $B_{l j}$, which is also an edge. Then we label them by using Lemma 5 . 
Step 7: If a block $B_{k-1, i}$ at level $k-1$ is a triangle, let its adjacent block at level $k$ be $B_{k j}$ and assume that it is also a cycle of finite length. If each vertex of $B_{k j}$ connected with a cycle triangle, one of them say $B_{k+1, p}$ at level $k+1$, then label the block by using Lemma 14 .

end MINLV

\subsection{Time complexity}

The correctness of the algorithm follows from the lemmas proved in the paper.

Theorem 2 The time complexity of the algorithm MINLV is $O(n)$.

Proof. The blocks and cutvertices of any graph can be computed in $O(m+n)$ time [19]. For the cactus graph $m=O(n)$. Hence step 1 of algorithm MINLV takes $O(n)$ time.

The time complexity to label the vertices of a block of size $m_{1}$ is $O\left(m_{1}\right)$. Step 4 labels the vertices of the blocks which are at level 1 of $G^{\prime}$. If the number of vertices of all blocks of this level is $m_{2}$, then the time complexity for step 4 is $O\left(m_{2}\right)$. That is the time complexity depends upon the number of vertices of the whole graph. Since the number of vertices of the entire graph is $O(n)$, the time complexity of the algorithm is $O(n)$.

Thus the $L(2,1)$-labelling of any cactus graph can be done using $O(n)$ time. 


\section{References}

[1] S S Adams, J Cass, M Tesch, D Sakai Troxell and C Wheeland, "The minimum span of $L(2,1)$ - labeling of certain generalized Petersen graphs," Disc. Appl. Math., vol. 155, pp. 1314-1325, 2007.

[2] G J Chang and David Kuo, "The $L(2,1)$-labelling problem on graphs," SIAM J. Discrete Math., vol. 9, pp. 309-316, 1996.

[3] G J Chang and C Lu, "Distance two labelling of graphs," European J. Combin., vol. 24, pp. 53-58, 2003.

[4] S H Chiang and J H Yan, "On $L(d, 1)$ - labeling of Cartesian product of a path," Discrete Appl. Math., vol. 156, pp. 2867-2881, 2008.

[5] J P Georges, D W Mauro and M A Whittlesey, "Relating path covering to vertex labelings with a condition at distance two," Discrete Math., vol. 135, pp. 103-111, 1994.

[6] J Georges and D W Mauro, "On the criticality of graphs labelled with a condition at distance two," Congr. Numer., vol. 101, pp. 3349, 1994.

[7] J Georges and D W Mauro, “On generalized Petersen graphs labelled with a condition at distance two," Discrete Math., vol. 259, pp. 311-318, 2002.

[8] J Georges and D W Mauro, On regular graphs optimally labelled with condition at distance two, SIAM J. Discrete Math., vol. 17, pp. 320-331, 2003.

[9] J Georges, D W Mauro and M I Stein, "Labelling products of complete graphs with a condition at distance two," SIAM J. Discrete Math., vol. 14, pp. 28- 35, 2000. 
[10] J Georges, D W Mauro and M Whittlesey, "Relating path covering to vertex labelling with a condition of distance two," Discrete Math., vol. 135, pp. 103-111, 1994.

[11] D Goncalves, "On the $L(p, 1)$-labelling of graphs, In: EuroCom 2005, Discrete Math. and Theoretical Comput. Sci. Proc., vol. AE, pp. 81-86, 2005.

[12] J R Griggs and R K Yeh, "Labelling graphs with a condition at distance two," SIAM J. Discrete Math., vol. 5, pp. 586-595, 1992.

[13] W K Hale, "Frequency assignment: Theory and applications," Proc. IEEE, vol. 68, pp. 1497-1514, 1980.

[14] J Van den Heuvel and S McGuinnes, "Coloring the square of a planar graph," J. Graph Theory, vol. 42, pp. 110-124, 2003.

[15] K Jonas, "Graph coloring analogue with a condition at distance two: L(2,1)-labellings and list $\lambda$-labellings," Ph.D. Thesis, University of South Carolina, Columbia, 1993.

[16] D Kral and R Skrekovski, "A theorem on channel assignment problem," SIAM J. Discrete Math., vol. 16, pp. 426-437, 2003.

[17] D D F Liu and R K Yeh, “On distance-two labellings of graphs," Ars Combin., vol. 47, pp. 13-22, 1997.

[18] M Molloy and M R Salavatipour, "A bound on the chromatic number of the square of a planar graph," J. Combin. Theory, Ser. B, vol. 94, pp. 189-213, 2005.

[19] E M Reingold, J Nivergent and N Deo, Combinatorial algorithms: theory and practice, New Jersy: Prentice Hall, Inc., 1977.

[20] D Sakai, "Labelling chordal graphs: Distance two condition," SIAM J. Discret. Math., vol. 7, pp. 133-140, 1994.

[21] W F Wang and K W Lih, "Labelling planar graphs with conditions on girth and distance two," SIAM J. Discrete Math., vol. 17, pp. 499-509, 2004. 
Nasreen Khan, Madhumangal Pal and Anita Pal ISSN 0975-3303

[22] M A Whittlesey, J P Georges and D W Mauro, "On the $\lambda$ number of $Q_{n}$ and related graphs," SIAM J. Discrete Math., vol. 8, pp. 499-506, 1995.

[23] R K Yeh, "Labelling graphs with a condition at distance two," $\mathrm{Ph}$. D. thesis, Department of Mathematics, University of South Carolina, Columbia, SC, 1990. 